Research Article

\title{
Plasma Levels of High-Mobility Group Box 1 during Peptide Vaccination in Patients with Recurrent Ovarian Cancer
}

\author{
Kayoko Waki, ${ }^{1}$ Kouichiro Kawano, ${ }^{2}$ Naotake Tsuda, ${ }^{2}$ Kimio Ushijima, ${ }^{2}$ Kyogo Itoh, ${ }^{3}$ and \\ Akira Yamada ${ }^{1}$ \\ ${ }^{1}$ Cancer Vaccine Development Division, Research Center for Innovative Cancer Therapy, Kurume University, Kurume, Japan \\ ${ }^{2}$ Department of Obstetrics and Gynecology, Kurume University School of Medicine, Kurume, Japan \\ ${ }^{3}$ Cancer Vaccine Center, Kurume University, Kurume, Japan
}

Correspondence should be addressed to Akira Yamada; akiymd@med.kurume-u.ac.jp

Received 6 February 2017; Accepted 5 March 2017; Published 27 April 2017

Academic Editor: Shahab Uddin

Copyright ( 2017 Kayoko Waki et al. This is an open access article distributed under the Creative Commons Attribution License, which permits unrestricted use, distribution, and reproduction in any medium, provided the original work is properly cited.

\begin{abstract}
High-mobility group box 1 (HMGB1) is a nuclear protein that is known to be secreted into extracellular fluids from injured cells, activated macrophages, and tumor cells. The clinical correlation of circulating HMGB1 levels with various diseases including cancer has been reported. However, there is no information on HMGB1 levels in cancer patients treated with peptide vaccination. In the present study, we investigated the plasma levels of HMGB1 during personalized peptide vaccination in patients with recurrent ovarian cancer. Frozen plasma samples of 39 patients from previously conducted clinical trials were used in this study. HMGB1 levels were decreased after the 1st cycle of vaccination from their prevaccination levels. However, no correlation was observed between HMGB1 and overall survival (OS). The correlation between plasma HMGB1 levels and other biomarker levels was further analyzed by scatter plot, revealing that HMGB1 levels after the 1st cycle of vaccination were significantly correlated with myeloid-derived suppressor cell (MDSC) frequency after the 1st cycle of vaccination $(r=0.357, p=0.032)$. Chi-square test showed that epitope spreading was significantly related with changes of HMGB1 $(p=0.030)$. These results suggest that plasma HMGB1 is a possible biomarker for cancer vaccine therapy, although direct correlation with OS has not been obtained. This trial is registered with Clinical Trial Registry under trial numbers UMIN000003083 and UMIN000001482.
\end{abstract}

\section{Introduction}

Ovarian cancer is one of the most common cancers in women worldwide. The standard first-line treatment is chemotherapy with platinum and taxane agents, and the majority of cases respond well to this treatment $[1,2]$. However, most cases experienced relapse and became resistant to platinum and subsequent chemotherapeutic agents [2]. Recent studies have demonstrated that immune checkpoint blockade therapy is very effective for the treatment of melanoma and non-small-cell lung cancer, although these dramatic clinical effects were observed in only $20-30 \%$ of patients [3-5]. Promising results of immune checkpoint blockade therapy have also been reported in ovarian cancer [6].

We previously reported a clinical study of personalized peptide vaccination (PPV) for recurrent ovarian cancer patients, in which the vaccine peptides were selected from a peptide panel consisting of 31 cytotoxic T-lymphocyte(CTL-) epitope peptides based on preexisting host immunity before vaccination [7]. In that study, we analyzed the relationship of several immune-related or inflammation-related biomarkers with the prognosis of patients and circulating lymphocyte frequency at prevaccination, and epitope spreading after the 1st cycle of vaccination was found to be significantly prognostic of overall survival (OS) [7]. However, other biomarkers were not prognostic or predictive. Therefore, the exploration of new biomarkers for cancer vaccine will be important.

High-mobility group box 1 (HMGB1) is a nuclear protein that is known to be secreted into plasma and other extracellular fluids from injured cells, activated macrophages, and tumor cells [8-11]. Circulating HMGB1 levels have been clinically and pathologically correlated with various diseases, including cancer [12]. However, there is no information on 


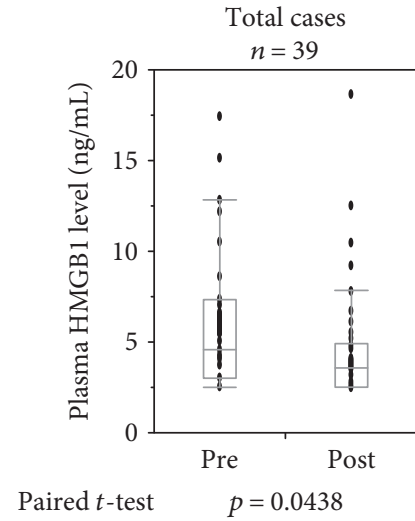

(a)

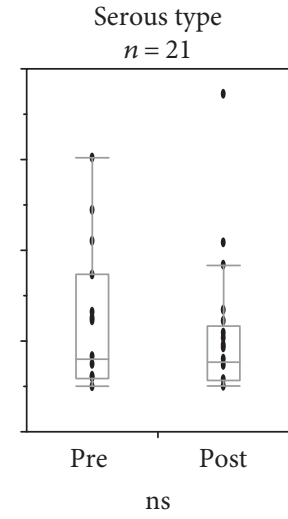

(b)

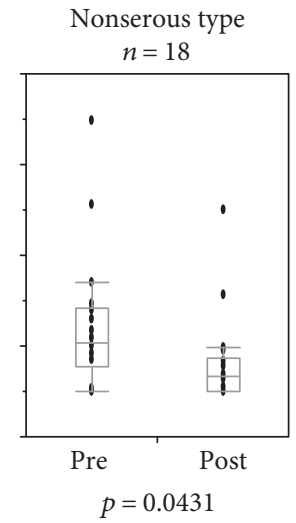

(c)

FIgURe 1: Plasma levels of HMGB1 during peptide vaccination. Plasma HMGB1 levels of pre- and post-1st cycle vaccination samples of the total 39 cases (a), the 21 serous-type cases (b), and the 18 non-serous-type cases (c) are shown. ns: Not significant.

HMGB1 levels in cancer patients treated with peptide vaccination. In the present study, therefore, we investigated the plasma levels of HMGB1 during PPV in patients with recurrent ovarian cancer.

\section{Materials and Methods}

2.1. Plasma Samples. Frozen plasma samples that had been collected from 39 patients with recurrent ovarian cancer who were treated with PPV in previously conducted clinical trials and stored at $-80^{\circ} \mathrm{C}$ were used in this study. The protocols of the clinical trials, including protocols for the measurement of plasma biomarkers, were approved by the Kurume University Ethics Committee and registered with the UMIN Clinical Trial Registry under trial numbers UMIN000003083 and UMIN000001482. One vaccination cycle consisted of once a week vaccination for six consecutive weeks.

Plasma HMGB1 levels were measured by using an HMGB1 ELISA kit II (Shino-Test, Sagamihara, Japan) according to the manufacturer's instructions. The detection limit was $2.5 \mathrm{ng} / \mathrm{mL}$ in our system.

2.2. Statistical Analysis. The statistical analyses were performed using JMP Pro version 12 software (SAS Institute, Cary, NC). Changes in the HMGB1 levels between prevaccination and postvaccination samples were analyzed by paired $t$-test. The correlation between HMGB1 levels and OS was analyzed by log-rank test. The comparisons between plasma HMGB1 levels and other biomarkers were analyzed by $t$-test and chi-square test.

\section{Results}

3.1. Plasma Levels of HMGB1 during Peptide Vaccination. Frozen plasma samples of 39 patients with recurrent ovarian cancer who had been treated with PPV in clinical trials were used in this study. The HMGB1 levels after the 1st cycle of vaccination (postvaccination) $(4.63 \pm 3.25 \mathrm{ng} / \mathrm{mL})$ were significantly lower than the prevaccination values (5.96 $\pm 3.69 \mathrm{ng} / \mathrm{mL}) \quad(p=0.043) \quad$ (Figure 1). The 39 patients consisted of patients with serous adenocarcinoma $(n=21)$, endometrioid carcinoma $(n=7)$, clear-cell carcinoma $(n=3)$, mucinous adenocarcinoma $(n=3)$, squamous cell carcinoma $(n=1)$, undifferentiated malignant tumor $(n=2)$, and others $(n=2)$. The patients were divided into two groups, that is, serous type $(n=21)$ and nonserous type $(n=18)$, for subgroup analyses. Prevaccination HMGB1 levels of the serous and nonserous types were, respectively, $5.87 \pm 3.69 \mathrm{ng} / \mathrm{mL}$ and $6.07 \pm 3.79 \mathrm{ng} / \mathrm{mL}$, and those of the postvaccination group were $5.16 \pm 3.75 \mathrm{ng} / \mathrm{mL}$ and $4.00 \pm 2.50 \mathrm{ng} / \mathrm{mL}$, respectively. Therefore, the significant decrease in HMGB1 levels after the 1st cycle of vaccination found in the overall group of 39 patients was confirmed in the non-serous-type group ( $n=18, p=0.043$ ) but not in the serous type group.

3.2. Relationship between HMGB1 Levels and Overall Survival. Patients were divided into high-HMGB1 group and low-HMGB1 group according to whether their pre- or postvaccination levels of plasma HMGB1 were higher or lower than the median value. Patients were also divided into a decreased or a not-decreased group according to the changes between their pre- and postvaccination values, and the correlations between these groups and OS were analyzed by Kaplan-Meier plot analysis (Figure 2). No significant correlations were found between the plasma HMGB1 levels and OS in either the pre- or postvaccination samples (Figures 2(a) and 2(b), resp.). Changes of plasma HMGB1 levels were similarly not a significant prognostic factor in OS (Figure 2(c)). It is noted that $20 \%$ of patients belonged to the low-HMGB1 prevaccination group and that $25 \%$ of the not-decreased group were long-term survivors with $>37$ months of OS.

3.3. Comparison between Plasma HMGB1 Levels and Other Biomarkers. In our previous analysis of this clinical study, we reported that epitope spreading after the 1st cycle of vaccination was a significant prognostic factor correlated to OS [7]. The other markers used in the previous study were as follows: IgG and CTL responses to the vaccinated peptides, frequency of circulating myeloid-derived suppressor cells (MDSCs), plasma levels of serum amyloid A (SAA), 


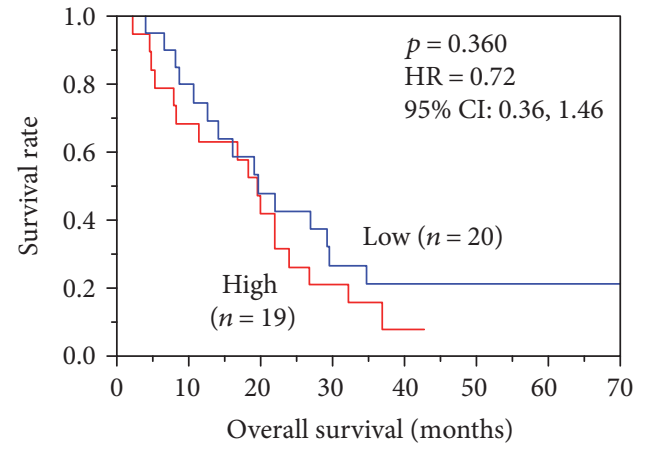

(a)

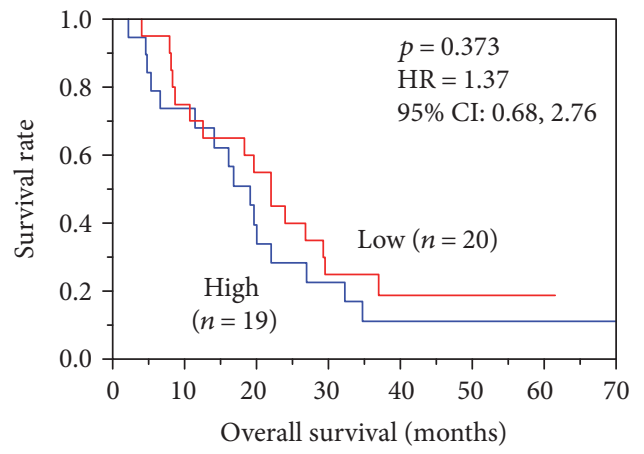

(b)

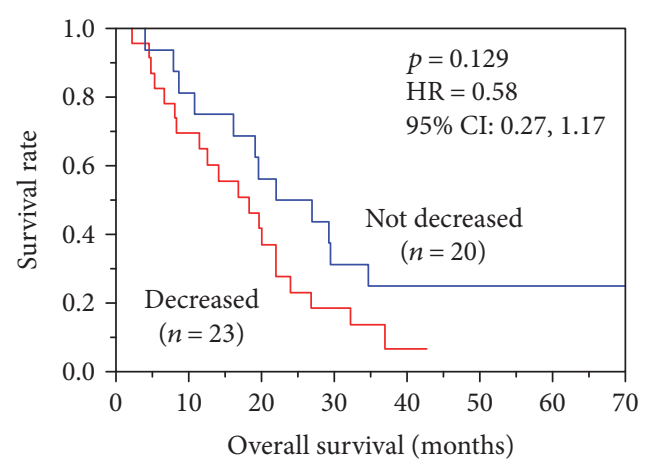

(c)

Figure 2: The relationship between plasma HMGB1 levels and overall survival (OS) was analyzed by Kaplan-Meier plot analysis. Patients $(n=39)$ were divided into high and low plasma HMGB1 groups based on the (a) pre- and (b) postvaccination levels, and the correlation between these subgroups and OS was analyzed. (c) Patients $(n=39)$ were also divided into decreased and not-decreased groups based on the changes of plasma HMGB1 between the prevaccination and postvaccination samples, and the relation between these subgroups and OS was plotted.
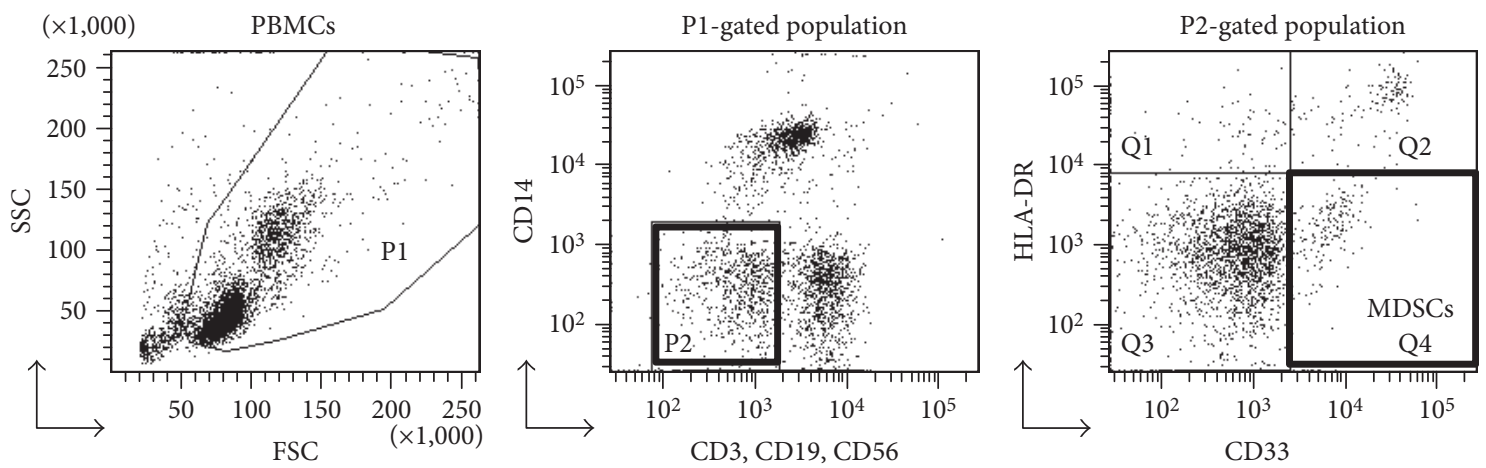

FIGURE 3: Gating strategy for the identification of MDSCs in the peripheral blood of patients. PBMCs were stained with anti-CD3-FITC, antiCD19-FITC, anti-CD56-FITC, anti-CD33-APC, anti-HLA-DR-PE/Cy7, and anti-CD14-APC/Cy7 Abs. In the cell subset negative for the lineage markers (CD3, CD19, CD56, and CD14) and HLA-DR, MDSCs were identified as CD33 ${ }^{+}$.

C-reactive protein (CRP), and interleukin-6 (IL-6). MDSCs in the peripheral blood were defined as $\mathrm{CD}_{3} 3^{+} \mathrm{CD}^{-} \mathrm{CD} 14^{-}$ CD19- ${ }^{-}$CD56 $6^{-}$HLA-DR ${ }^{-}$cells [7]. Gating strategy for the identification of MDSCs is shown in Figure 3. HMGB1 levels and previously measured data of the biomarker levels are shown in Table 1. Precise data of IgG and CTL responses and epitope spreading were previously published, and thus, these results are only summarized in Table 1. Correlations between the plasma HMGB1 levels and the other biomarker levels were analyzed by scatter plot. The postvaccination HMGB1 levels were significantly correlated with the postvaccination MDSC frequency $(r=0.357, p=0.032)$ but not with the postvaccination values of SAA, CRP, or IL-6 (Figure 4). Prevaccination values or changes between the pre- and postvaccination values of $\mathrm{HMGB} 1$ were not correlated with the corresponding values of SAA, CRP, or IL-6 (data not shown).

Changes of plasma HMGB1 levels were categorized into three groups, an increase, a decrease, and a no-change group. 


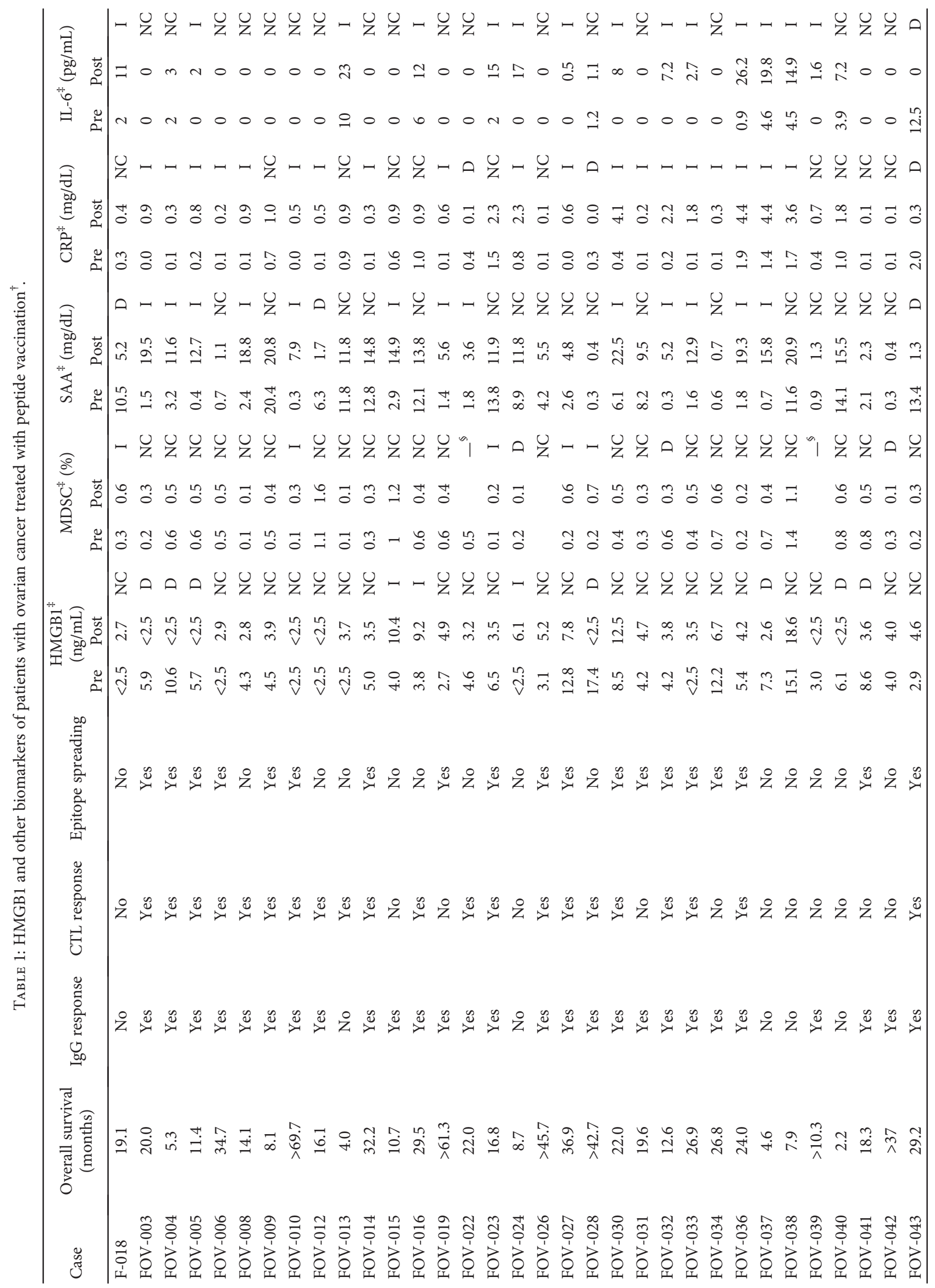




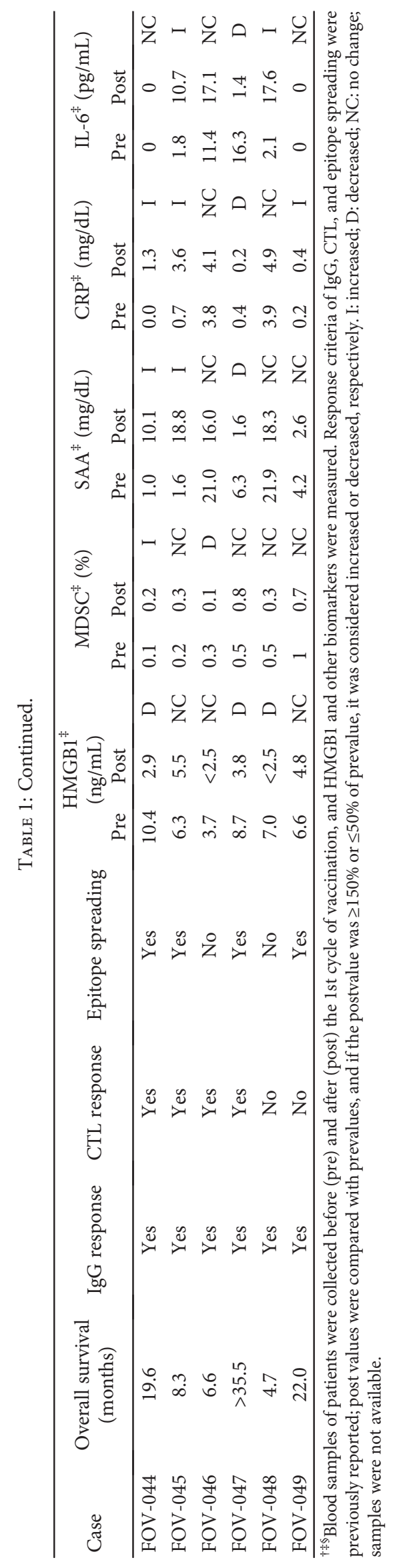



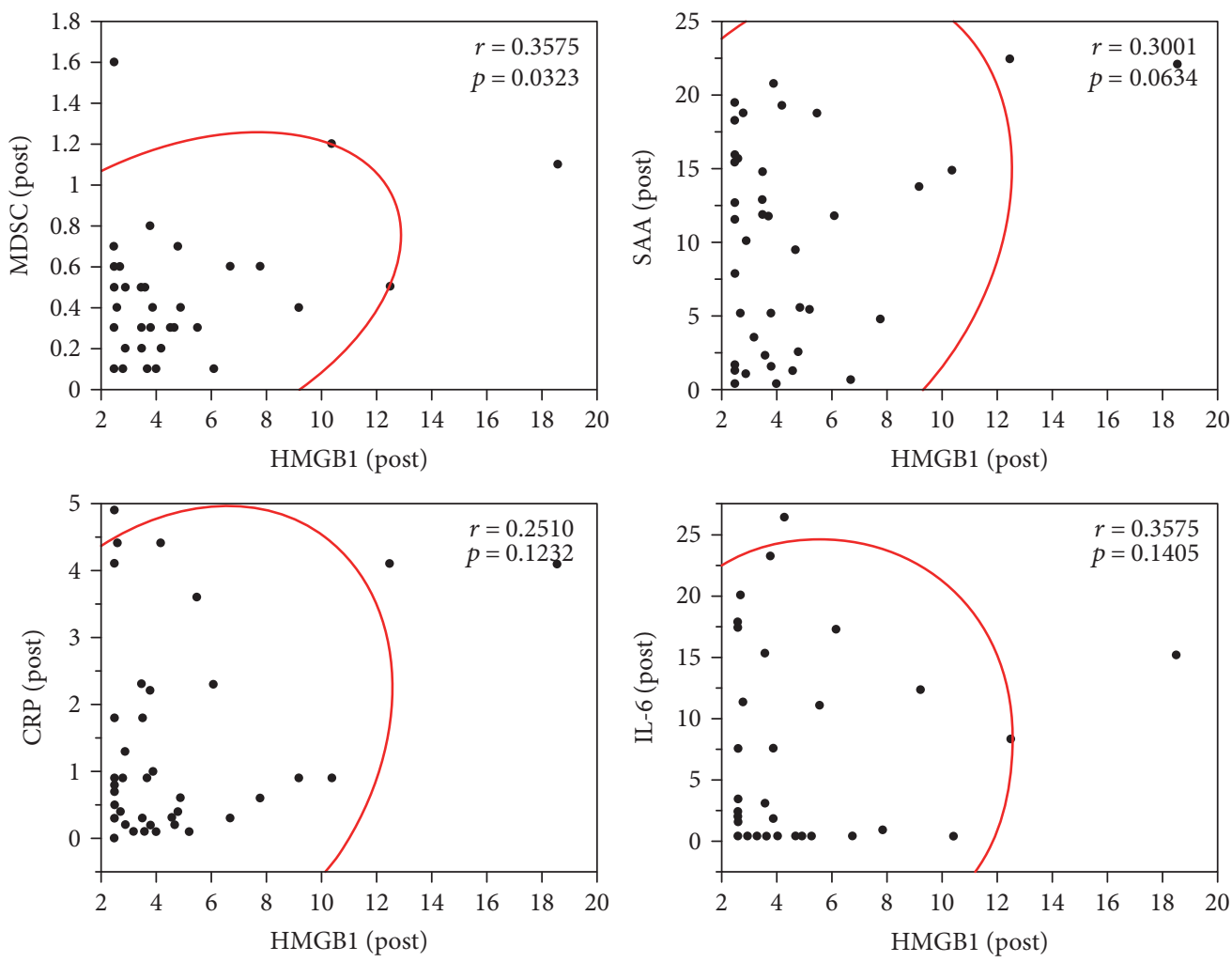

FIgURE 4: Correlations between postvaccination plasma HMGB1 levels and postvaccination levels of MDSC frequency, SAA, CRP, or IL-6 were analyzed by scatter plot.

Similarly, changes in each of the other biomarkers were categorized as simply "yes" or "no" (Table 2). The proportions of the categories of HMGB1 and the other biomarkers were compared by chi-square test. The results showed that epitope spreading was significantly related with changes of HMGB1 $(p=0.030)$ and that IgG and CTL responses, MDSC, SAA, CRP, and IL-6 were independent of HMGB1.

\section{Discussion}

HMGB1 is a representative member of the damage-associated molecular patterns (DAMPs), also called alarmins, which are molecules released from necrotic cells as intrinsic danger signals which induce inflammation and trigger innate immunity [8]. Toll-like receptor (TLR) 2 or 4 and receptor for advanced glycation end products (RAGE) are known to be receptors of HMGB1 [13, 14]. In addition to the role of initiator of innate immunity, HMGB1 also exerts an immunosuppressive effect through the $\mathrm{T}$ cell immunoglobulin domain and mucin domain 3 (TIM3), an immune checkpoint molecule on activated $\mathrm{T}$ cells [15]. Recently, we found that HMGB1 inhibitors, such as glycyrrhizin, exhibited an immunopotentiating effect in combination with an innate immunity receptor-related adjuvant in a murine peptide vaccine model [16]. These facts indicate that HMGB1 has two opposite functions, that is, it both initiates and suppresses immunity.

Circulating HMGB1 levels have been clinically and pathologically correlated with various diseases, including cancer $[12,17]$. High levels of HMGB1 have been correlated with worsened disease outcome in most types of cancers, including colorectal cancer, bladder cancer, and hepatocellular carcinoma [12]. However, the opposite results were also obtained in some cancers - namely, high levels of HMGB1 were correlated with better prognosis in patients with esophageal cancer and gastric cancer [12]. These results were obtained from patients in various clinical settings, although none were undergoing immune therapy. In fact, to our knowledge, there has been only one report on the HMGB1 levels of patients treated with immunotherapy: Gebhardt et al. [18] reported the possibility of circulating HMGB1 as a novel predictive marker for melanoma patients who may benefit from ipilimumab (anti-CTLA-4 antibody) therapy. In the present study, therefore, we investigated the plasma levels of HMGB1 in patients with ovarian cancer treated with peptide vaccination. The presence of circulating HMGB1 in patients with ovarian cancer has been reported, and the levels were significantly higher than those in patients with benign ovarian tumors or healthy donors, and the levels in recurrent patients were higher than those in nonrecurrent patients $[19,20]$.

In the present study, plasma HMGB1 levels were decreased after the 1st cycle of vaccination (Figure 1). The most plausible mechanism of this decrease in HMGB1 is that the tumor mass was reduced by vaccination. However, in most of the cases studied, the best clinical response was progressive disease [7], and thus, it was hard to confirm that such a mechanism was operative. Further accumulation and analysis of biomarkers may clarify whether this mechanism plays a role. Approximately $20-25 \%$ of patients belonging to the group with low prevaccination HMGB1 or the notdecreased group were long-term survivors with $>37$ months 
TABLE 2: Comparison of HMGB1 and other biomarkers.

\begin{tabular}{|c|c|c|c|c|c|c|c|c|c|c|c|c|c|c|}
\hline \multirow{3}{*}{ HMGB1 increase (I) } & \multicolumn{2}{|c|}{$\begin{array}{c}\operatorname{IgG} \\
\text { response } \\
n=39\end{array}$} & \multicolumn{2}{|c|}{$\begin{array}{c}\mathrm{CTL} \\
\text { response } \\
n=39\end{array}$} & \multicolumn{2}{|c|}{$\begin{array}{c}\text { Epitope } \\
\text { spreading } \\
n=39\end{array}$} & \multicolumn{2}{|c|}{$\begin{array}{c}\text { MDSC } \\
\text { increase } \\
n=37\end{array}$} & \multicolumn{2}{|c|}{$\begin{array}{c}\text { SAA } \\
\text { increase } \\
n=39\end{array}$} & \multicolumn{2}{|c|}{$\begin{array}{c}\text { CRP } \\
\text { increase } \\
n=39\end{array}$} & \multicolumn{2}{|c|}{$\begin{array}{c}\text { IL-6 } \\
\text { increase } \\
n=39\end{array}$} \\
\hline & & $n$ & & $n$ & & $n$ & & $n$ & & $n$ & & $n$ & & $n$ \\
\hline & Yes & 2 & Yes & 1 & Yes & 0 & Yes & 0 & Yes & 1 & Yes & 1 & Yes & 2 \\
\hline & No & 1 & No & 2 & No & 3 & No & 3 & No & 2 & No & 2 & No & 1 \\
\hline \multirow[t]{2}{*}{ HMGB1 no change (NC) } & Yes & 23 & Yes & 18 & Yes & 17 & Yes & 4 & Yes & 9 & Yes & 16 & Yes & 11 \\
\hline & No & 3 & No & 8 & No & 9 & No & 20 & No & 17 & No & 10 & No & 15 \\
\hline \multirow[t]{2}{*}{ HMGB1 decrease (D) } & Yes & 8 & Yes & 6 & Yes & 6 & Yes & 2 & Yes & 5 & Yes & 5 & Yes & 3 \\
\hline & No & 2 & No & 4 & No & 4 & No & 8 & No & 5 & No & 5 & No & 7 \\
\hline \multicolumn{15}{|l|}{$p$ value $^{\dagger}$} \\
\hline I versus not I $(\mathrm{NC}+\mathrm{D})$ & & 0.3698 & & 0.2475 & & $0.0307^{*}$ & & 0.4267 & & 0.8493 & & 0.4015 & & 0.3473 \\
\hline \multirow[t]{4}{*}{$\mathrm{D}$ versus not $\mathrm{D}(\mathrm{NC}+\mathrm{I})$} & & 0.639 & & 0.7538 & & 0.939 & & 0.7039 & & 0.3844 & & 0.6355 & & 0.4111 \\
\hline & & & & & & & & $\begin{array}{l}\text { SC } \\
\text { ease }\end{array}$ & & $\begin{array}{l}\mathrm{AA} \\
\text { rease }\end{array}$ & & $\begin{array}{l}\mathrm{RP} \\
\text { rease }\end{array}$ & & $\begin{array}{l}-6 \\
\text { rease }\end{array}$ \\
\hline & & & & & & & & 37 & & $=39$ & & $=39$ & & $=39$ \\
\hline & & & & & & & & $n$ & & $n$ & & $n$ & & $n$ \\
\hline \multirow[t]{2}{*}{ HMGB1 increase (I) } & & & & & & & Yes & 1 & Yes & 0 & Yes & 0 & Yes & 0 \\
\hline & & & & & & & No & 2 & No & 3 & No & 3 & No & 3 \\
\hline \multirow[t]{2}{*}{ HMGB1 no change (NC) } & & & & & & & Yes & 3 & Yes & 3 & Yes & 2 & Yes & 1 \\
\hline & & & & & & & No & 21 & No & 23 & No & 24 & No & 25 \\
\hline \multirow[t]{2}{*}{ HMGB1 decrease (D) } & & & & & & & Yes & 0 & Yes & 1 & Yes & 2 & Yes & 1 \\
\hline & & & & & & & No & 10 & No & 9 & No & 8 & No & 9 \\
\hline \multicolumn{15}{|l|}{$p$ value } \\
\hline I versus not I $(\mathrm{NC}+\mathrm{D})$ & & & & & & & & 0.19 & & 0.5422 & & 0.5422 & & 0.6751 \\
\hline $\mathrm{D}$ versus not $\mathrm{D}(\mathrm{NC}+\mathrm{I})$ & & & & & & & & 0.1975 & & 0.9753 & & 0.2389 & & 0.418 \\
\hline
\end{tabular}

${ }_{{ }^{*}} p$ values of chi-square test are shown; statistically significant.

of OS (Figure 2). These results may suggest that HMGB1 could be used as a predictive and/or prognostic marker of peptide vaccination. We also found that postvaccination plasma HMGB1 levels were significantly correlated with the post-MDSC frequency (Figure 3). It is known that chronic inflammation facilitates malignancy by inducing the accumulation and increasing the potency of MDSCs [21]. HMGB1 is an initiator of inflammation and has been reported to play a role in the development of MDSCs [22]. CCL2, a member of C-C chemokine family, is known as a major cytokine for the recruitment of myeloid cells including MDSCs from the bone marrow to cancer tissues [23, 24], and induction of CCL2 in various cells by HMGB1 has also been reported $[25,26]$. These facts support our findings. Epitope spreading has been reported as an immune responserelated prognostic marker of good outcome in our previous analysis of this clinical trial [7]. Epitope spreading was not observed in the cases with increased HMGB1 in this study. Thus, epitope spreading might be due to the suppression of immune responses by MDSCs induced by HMGB1.

\section{Conclusion}

Our present data suggest that plasma HMGB1 may be a predictive and/or prognostic marker for cancer vaccine therapy, although at the present time, there remains no direct correlation with OS. Future clinical studies on the circulating HMGB1 levels in large numbers of patients will be needed to clarify the usefulness of HMGB1 in immunotherapy.

\section{Conflicts of Interest}

The authors declare that there is no conflict of interest regarding the publication of this paper.

\section{Acknowledgments}

This study was supported by MEXT-Supported Program for the Strategic Research Foundation at Private University.

\section{References}

[1] S. Komiyama, H. Katabuchi, M. Mikami et al., "Japan Society of Gynecologic Oncology guidelines 2015 for the treatment of ovarian cancer including primary peritoneal cancer and fallopian tube cancer," International Journal of Clinical Oncology, vol. 21, no. 3, pp. 435-446, 2016.

[2] O. W. Foley, J. A. Rauh-Hain, and M. G. del Carmen, "Recurrent epithelial ovarian cancer: an update on treatment," Oncology, vol. 27, no. 4, pp. 288-294, 2013, 298.

[3] J. M. Redman, G. T. Gibney, and M. B. Atkins, "Advances in immunotherapy for melanoma," BMC Medicine, vol. 14, no. 1, p. 20, 2016. 
[4] J. Brahmer, K. L. Reckamp, P. Baas et al., "Nivolumab versus docetaxel in advanced squamous-cell non-small-cell lung cancer," The New England Journal of Medicine, vol. 373, no. 2, pp. 123-135, 2015.

[5] H. Borghaei, L. Paz-Ares, L. Horn et al., "Nivolumab versus docetaxel in advanced nonsquamous non-small-cell lung cancer," The New England Journal of Medicine, vol. 373, no. 17, pp. 1627-1639, 2015.

[6] J. Hamanishi, M. Mandai, and I. Konishi, "Immune checkpoint inhibition in ovarian cancer," International Immunology, vol. 28, no. 7, pp. 339-348, 2016.

[7] K. Kawano, N. Tsuda, S. Matsueda et al., "Feasibility study of personalized peptide vaccination for recurrent ovarian cancer patients," Immunopharmacology and Immunotoxicology, vol. 36, no. 3, pp. 224-236, 2014.

[8] P. Scaffidi, T. Misteli, and M. E. Bianchi, "Release of chromatin protein HMGB1 by necrotic cells triggers inflammation," Nature, vol. 418, no. 6894, pp. 191-195, 2002.

[9] S. Gardella, C. Andrei, D. Ferrera et al., "The nuclear protein HMGB1 is secreted by monocytes via a non-classical, vesiclemediated secretory pathway," EMBO Reports, vol. 3, no. 10, pp. 995-1001, 2002.

[10] T. Bonaldi, F. Talamo, P. Scaffidi et al., "Monocytic cells hyperacetylate chromatin protein HMGB1 to redirect it towards secretion," The EMBO Journal, vol. 22, no. 20, pp. 5551-5560, 2003.

[11] D. Tang, Y. Shi, R. Kang et al., "Hydrogen peroxide stimulates macrophages and monocytes to actively release HMGB1," Journal of Leukocyte Biology, vol. 81, no. 3, pp. 741-747, 2007.

[12] J. Fucikova, I. Moserova, L. Urbanova et al., "Prognostic and predictive value of DAMPs and DAMP-associated processes in cancer," Frontiers in Immunology, vol. 6, p. 402, 2015.

[13] J. S. Park, D. Svetkauskaite, Q. He et al., "Involvement of toll-like receptors 2 and 4 in cellular activation by high mobility group box 1 protein," The Journal of Biological Chemistry, vol. 279, no. 9, pp. 7370-7377, 2004.

[14] I. E. Dumitriu, P. Baruah, B. Valentinis et al., "Release of high mobility group box 1 by dendritic cells controls T cell activation via the receptor for advanced glycation end products," Journal of Immunology, vol. 174, no. 12, pp. 7506-7515, 2005.

[15] S. Chiba, M. Baghdadi, H. Akiba et al., "Tumor-infiltrating DCs suppress nucleic acid-mediated innate immune responses through interactions between the receptor TIM-3 and the alarmin HMGB1," Nature Immunology, vol. 13, no. 9, pp. 832-842, 2012.

[16] K. Waki and A. Yamada, "Blockade of high mobility group box 1 (HMGB1) augments anti-tumor T-cell response induced by peptide vaccination as a co-adjuvant," Cancer Science, vol. 107, no. 12, pp. 1721-1729, 2016.

[17] R. Kang, R. Chen, Q. Zhang et al., "HMGB1 in health and disease," Molecular Aspects of Medicine, vol. 40, pp. 1-116, 2014.

[18] C. Gebhardt, A. Sevko, H. Jiang et al., "Myeloid cells and related chronic inflammatory factors as novel predictive markers in melanoma treatment with ipilimumab," Clinical Cancer Research, vol. 21, no. 24, pp. 5453-5459, 2015.

[19] Y. Li, J. Tian, X. Fu et al., "Serum high mobility group box protein 1 as a clinical marker for ovarian cancer," Neoplasma, vol. 61, no. 5, pp. 579-584, 2014.

[20] H. Wang, Z. Li, Y. Sun et al., "Relationship between highmobility group box 1 overexpression in ovarian cancer tissue and serum: a meta-analysis," OncoTargets and Therapy, vol. 8, pp. 3523-3531, 2015.

[21] S. Ostrand-Rosenberg and P. Sinha, "Myeloid-derived suppressor cells: linking inflammation and cancer," Journal of Immunology, vol. 182, no. 8, pp. 4499-4506, 2009.

[22] K. H. Parker, P. Sinha, L. A. Horn et al., "HMGB1 enhances immune suppression by facilitating the differentiation and suppressive activity of myeloid-derived suppressor cells," Cancer Research, vol. 74, no. 20, pp. 5723-5733, 2014.

[23] E. Chun, S. Lavoie, M. Michaud et al., "CCL2 promotes colorectal carcinogenesis by enhancing polymorphonuclear myeloid-derived suppressor cell population and function," Cell Reports, vol. 12, no. 2, pp. 244-257, 2015.

[24] A. L. Chang, J. Miska, D. A. Wainwright et al., "CCL2 produced by the glioma microenvironment is essential for the recruitment of regulatory $\mathrm{T}$ cells and myeloid-derived suppressor cells," Cancer Research, vol. 76, no. 19, pp. 56715682, 2016.

[25] X. Wu, Y. Mi, H. Yang, A. Hu, Q. Zhang, and C. Shang, "The activation of HMGB1 as a progression factor on inflammation esponse in normal human bronchial epithelial cells through RAGE/JNK/NF- $\kappa$ B pathway," Molecular and Cellular Biochemistry, vol. 380, no. 1-2, pp. 249-257, 2013.

[26] K. Shirasuna, K. Seno, A. Ohtsu et al., "AGEs and HMGB1 increase inflammatory cytokine production from human placental cells, resulting in an enhancement of monocyte migration," American Journal of Reproductive Immunology, vol. 75, no. 5, pp. 557-568, 2016. 


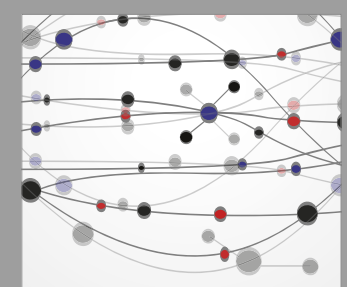

The Scientific World Journal
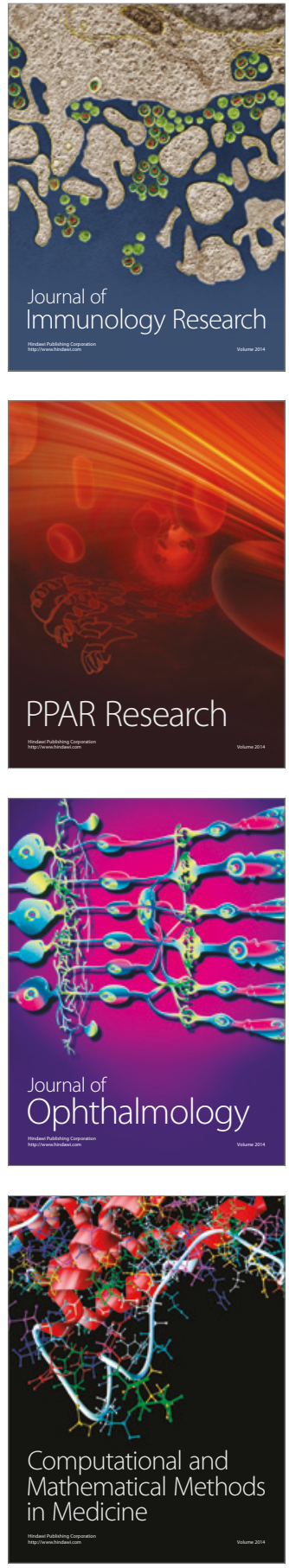

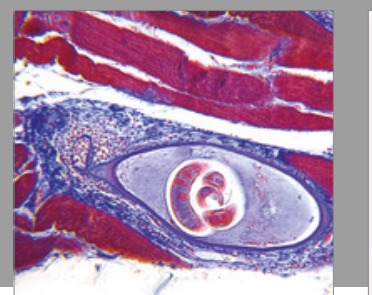

Gastroenterology Research and Practice
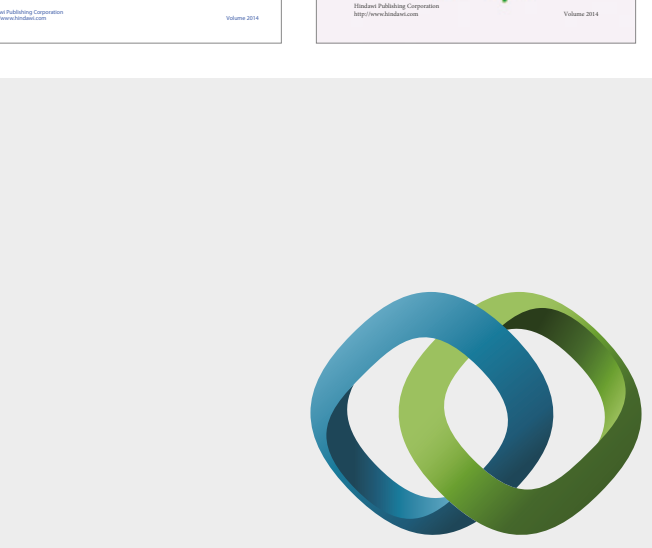

\section{Hindawi}

Submit your manuscripts at

https://www.hindawi.com
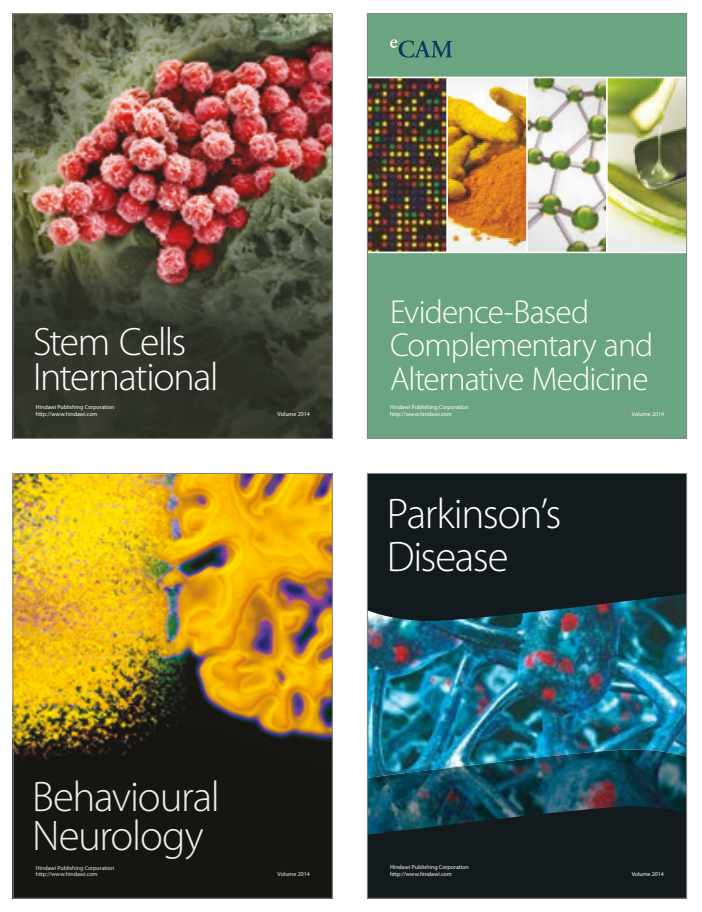
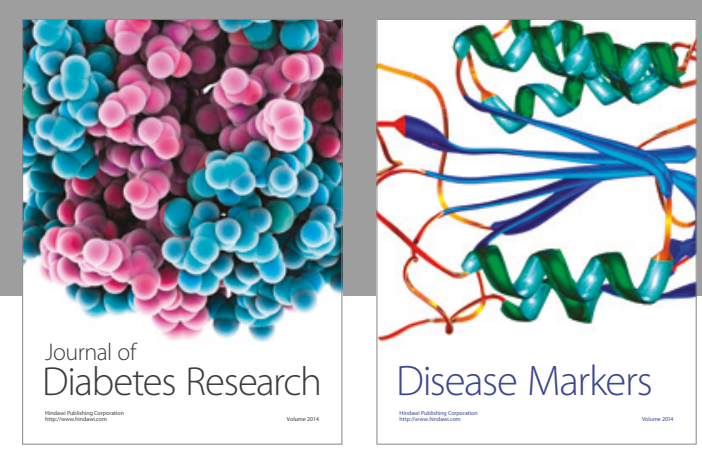

Disease Markers
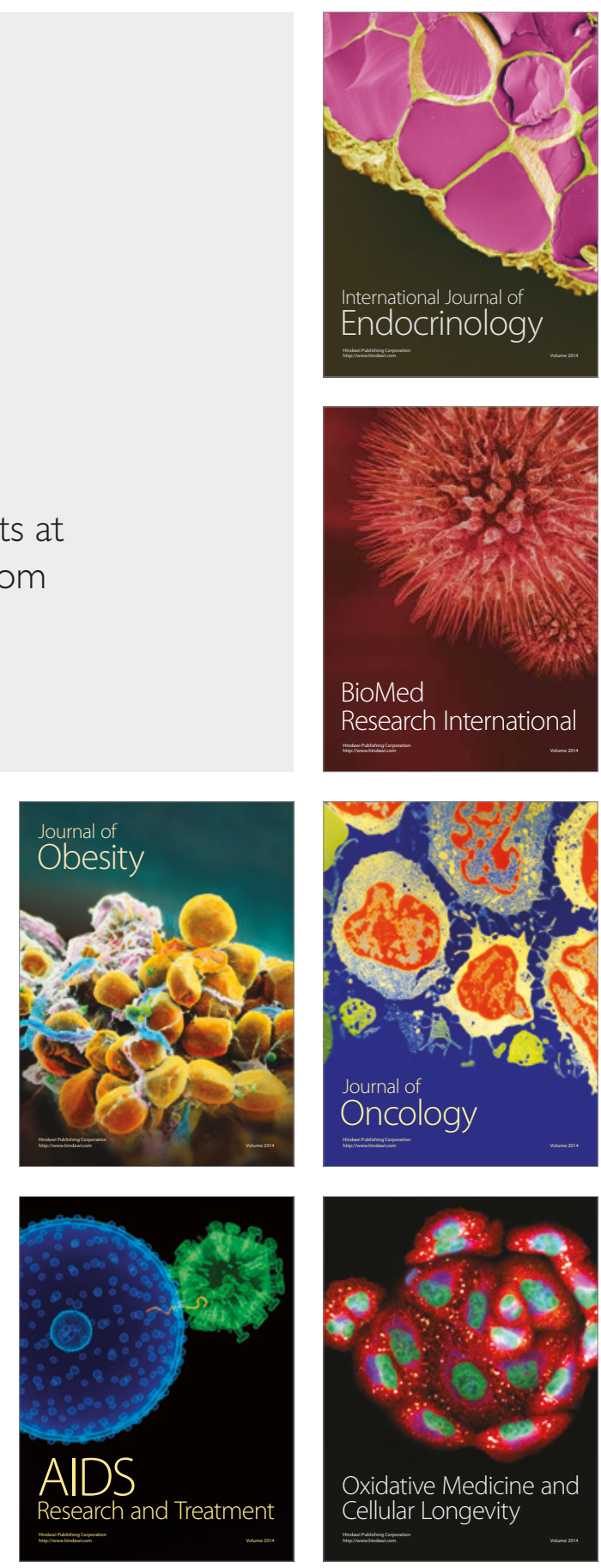